\title{
The theory and application of spectral risk measures in Vietnam
}

\author{
HO HONG HAI \\ Foreign Trade University - hai.ho@ftu.edu.vn \\ NGUYEN THI HOA \\ Sun Life Vietnam
}

ARTICLE INFO

Article history:

Received:

Oct. 17, 2016

Received in revised form:

July 04, 2017

Accepted:

Oct. 25,2017

Keywords:

Risk measure

Investment portfolio

Value-at-Risk

Expected shortfall

Spectral risk measures

Distortion theory

\section{ABSTRACT}

This paper aims to provide a new risk measure for portfolio management in Vietnam by incorporating investor's risk aversion into current risk measures such as value at risk (VaR) and expected shortfall (ES). This measure shares several desirable characteristics with the coherent risk measures, as illustrated in Artzner et al. (1997). In Vietnam, our study makes the first attempt to utilize distortion theory, instead of utility theory, to facilitate the adoption of risk aversion level in the popular risk measures. We find that spectral risk measure is more flexible and effective to different groups of risk-adverse investors, compared to the more monotonic and conventional VaR and ES measures. 


\section{Introduction}

In financial risk management, value at risk $(\mathrm{VaR})$ and expected shortfall (ES) are widely used as measures of risks. However, both VaR and ES fail to explicitly account for investor's risk appetite even though $\mathrm{VaR}$ is more suitable for risk-loving investor and ES matches riskneutral ones (GARP, 2016). As a result, VaR and ES are questionable measures for the predominant group of risk-adverse investors. For this group of investors, the later spectral risk measure (SRM) is more appropriate since it incorporates risk aversion into $\mathrm{VaR}$ and $\mathrm{ES}$ on the basis of the expected utility theory. However, Dowd et al. (2008) examined SRM based on this theory and found that the application of exponential utility theory in SRM implies a constant coefficient of absolute risk aversion and that relative risk aversion coefficient is an increasing function of investor's wealth. Such implication is in sharp contrast with the stylized facts that higher wealth endowment is associated with lower absolute risk aversion (i.e. the absolute risk aversion coefficient is the decreasing function of wealth) and constant coefficient of relative risk aversion (Copeland et al., 2005). Dowd et al. (2008) also illustrated the "bad behavior" of the power utility function in the weighting mechanism in which more weight is placed on lower loss and less weight on higher loss when the risk aversion coefficient is below one.

This paper addresses the uncanny behavior of risk aversion coefficient and exponential utility function by employing alternative functions rooted in the distortion theory. This approach is deemed more appropriate and advocated in recent studies (Sereda et al., 2010; Guegan \& Hassani, 2014). Since none of the study in the literature adopts this method for Vietnamese market, we embark on examining alternative spectral risk measures founded on fundamental distortion functions (e.g., dual-power, proportional hazard and Wang's (2000) measures). The study, therefore, makes an attempt to shed more light on the obscure application of distortion theory on financial risks measurement, especially in Vietnam.

\section{Literature review}

In modern portfolio theory, Markowitz (1952) suggested the mean-variance approach, which considers portfolio variance or standard deviation as a simple measure of risk. Under this approach, the portfolio optimization is conditional on the level of risk aversion, and the resulting choice is, however, inconsistent with the second order stochastic dominance in expected utility theory (Copeland et al., 2005). Thus, the portfolio standard deviation, despite being a popular choice, is an improper measure of risk (GARP, 2016).

Later, value at risk $(\mathrm{VaR})$ arose out of a Morgan's report in July 1993 and quickly became one of the standard risk measures in finance industry. The advantage of $\mathrm{VaR}$ is that it is especially effective with elliptical distribution of asset return (Grootveld \& Hallerbatch, 2004). However, the VaR approach requires fine-tuning via stress testing and scenarios analysis. This approach also fails to report losses in excess of VaR level, and it is inconsistent with the diversification effects due to the lack of subadditivity property when the asset return does not follow elliptical distribution.

To address this problem Artzner et al. (1997) and Artzner et al. (1999) proposed the coherent risk measures with four fundamental properties such as monotonicity, sub-additivity, linear homogeneity, and translational invariance. Tasche and Acerbi (2002) stated that Choquet expectation with a concave curve represents the 
coherent risk measure in general which is consistent with the rule of second order stochastic dominance.

Two prevalent coherent risk measure models that have recently been studied are expected shortfall (ES) and spectral risk measure (SRM). While ES is an average of the quantiles of a loss distribution, SRM is a weighted average of the quantiles, which connects the conventional risk measure to the individual's risk aversion. Accordingly, SRM weights grow proportionally with the level of user's risk aversion, implying that higher risk aversion is attached to higher level of risk and greater SRM value. SRM is also more appropriate than existing methods in that it is consistent with second order stochastic dominance in the expected utility theory (GARP, 2016). The advantages of the SRM approach have been demonstrated in other studies on a handful of issues such as setting capital requirements for banks or acquiring optimal riskreturn tradeoff (Acerbi, 2004), proposing capital allocation (Overbeck, 2004; Abad \& Iyengar, 2015; Adam et al., 2007), or setting margin requirements (Cotter and Dowd, 2006).

One of the major issues concerning the SRM approach is the choice of risk aversion function. Acerbi (2004) gauged just one risk aversion function derived from exponential utility theory. Dowd et al. (2008) examined the validity of exponential and power risk aversion and found that only the power level one satisfies the fundamental requirements of this approach. Recent studies suggested distortion theory as an alternative pathway, instead of expected utility theory, in the derivation of risk aversion function. Starting with Gzyl and Mayoral (2006) and Sriboonchitta et al. (2010) and subsequently Sereda et al. (2010) and Guegan and Hassani (2014), these papers aimed to introduce new asymmetric distortion risk measures based on the concept of risk for risk-adverse investors. Sereda et al (2010) proposed an asymmetric distortion risk measure based on power distortion function, which introduces different parameters on the left and right sides of the integral. However, Guegan and Hassani (2014) argued that it is insufficient to consider the same function with two different parameters. They indicated a convex distortion function for losses and a concave distortion function for gains and suggested the modified expected shortfall of the quantile using an Sinversed shaped distortion function.

This contemporary stream of research has not been widely tested. Therefore, this paper attempts to apply distortion theory and find empirical evidence of the new approach applicable to the Vietnam stock market.

\section{Research model}

\subsection{Spectral risk measure}

SRM shares several important properties of coherent risk measures. Assume that $\mathrm{X}$ and $\mathrm{Y}$ are the future values of two risky positions, a risk measure $\rho$ (.) is coherent if it satisfies the following four properties:

$\begin{array}{ll}\rho(\mathrm{X})+\rho(\mathrm{Y}) \geq \rho(\mathrm{X}+\mathrm{Y}) & \text { (Sub-additivity) } \\ \rho(\mathrm{tX})=\mathrm{t} \rho(\mathrm{X}) & \text { (Homogeneity) } \\ \rho(\mathrm{X}) \geq \rho(\mathrm{Y}), \text { if } \mathrm{X} \geq \mathrm{Y} & \text { (Monotonicity) } \\ \rho(\mathrm{X}+\mathrm{n})=\rho(\mathrm{X})-\mathrm{n} & \text { (Translational } \\ n>0, t>0 & \text { invariance) }\end{array}$

The sub-additivity property means that the total risk of a combined portfolio is equal to or less than the sum of the risk of individual assets, which is similar to the diversification effects. The absence of this property in $\mathrm{VaR}$ renders $\mathrm{VaR}$ ineffective in reducing idiosyncratic risks in portfolio management, and hence especially inappropriate for highly volatile market. In addition, VaR and expected shortfall (ES) fail to explicitly account for investor's risk aversion even though $\mathrm{VaR}$ is conventionally applicable 
for risk-loving investors while ES is more suitable for risk-neutral ones. To address this problem, spectral risk measure (SRM) embeds investor's risk aversion. It is defined as a weighted average of quantiles of return distribution of the portfolio.

$$
\begin{aligned}
& M_{\phi}=\int_{0}^{1} \phi(p) F_{X}^{-1}(p) d p \text { or } M_{\phi}= \\
& \int_{0}^{1} \phi(p) q_{X}(p) d p
\end{aligned}
$$

where:

$\mathrm{M}_{\phi}$ : the Spectral Risk Measure

$\mathrm{X}$ : the future value of the portfolio

$F_{X}^{-1}(p)$ or $q_{X}(p)$ : the quantile function of $\mathrm{X}$

$\phi(\mathrm{p})$ : weighting function or risk aversion function satisfying three following conditions:

$$
\begin{aligned}
& \phi(\mathrm{p}) \geq 0 \text { (non-negativity) } \\
& \int_{a}^{1} \phi(\mathrm{p}) d p=1 \text { (normalization) } \\
& \phi\left(\mathrm{p}_{1}\right) \geq \phi\left(\mathrm{p}_{2}\right), \text { for any } \mathrm{p}_{1} \geq \mathrm{p}_{2}{ }^{1} \text { or } \phi^{\prime}(\mathrm{p}) \geq 0
\end{aligned}
$$

(weak increasingness)

With the "weak increasingness" property, the weighting function assigns higher weight for higher loss and lower weight for lower loss, which represents the behavior of risk-adverse investors. However, when $\phi^{\prime}(p)=0$, the weight is indifferent to different loss levels, hence including risk-neutral investors in SRM set. In an attempt to strictly include risk aversion Dow et al. (2008) removed the equal sign in the third condition $\left(\phi^{\prime}(p) \geq 0\right)$ and replaced the "weak increasingness" with $\phi(\mathrm{p} 1)>\phi(\mathrm{p} 2)$ for any $\mathrm{p} 1$ $>$ p2 2 or $\phi^{\prime}(\mathrm{p})>0$.

Despite the modification on the "weak increasingness," the lack of explicit control for investor's risk aversion muddled Dow et al.'s (2008) explanations on "badly-behaved" cases in their adoption of the utility theory in spectral risk measures. Thus, the extra condition is added to control for risk aversion such that $\phi\left(\Upsilon_{1}\right) \geq \phi\left(\Upsilon_{2}\right)$, for $\Upsilon_{1} \geq \Upsilon_{2}$ or $\phi^{\prime}\left(\Upsilon_{1}\right) \geq 0$, with $\mathrm{p} \in[a, b]$ and $0<a<b<1$, where $\Upsilon$ reflects the investor's risk aversion.

\subsection{Distortion risk measure}

Distortion measures originated from the dual theory of choice under uncertainty, which advocated a risk measure based on a distortion function (Yaari, 1987). Denneberg (1994) developed the theory of integration to establish the connection between the concave distortion risk measures and spectral risk measures as follows:

$$
\begin{aligned}
& \rho_{f}(X)=M_{\phi}=\int_{-\infty}^{+\infty} x d(f \circ F)(x)= \\
& \int_{0}^{1} \phi(p) F_{X}^{-1}(p) d p
\end{aligned}
$$

where:

$\mathrm{M}_{\phi}$ : the notation of spectral risk measure

$\rho_{f}(\mathrm{X})$ : the general formula of a concave distortion function

$\int_{-\infty}^{+\infty} x d(f \circ F)(x)$ : the concave distortion function

$\int_{0}^{1} \phi(\mathrm{p}) F_{X}^{-1}(p) d p:$ the standard spectral risk measure

$$
f(p)=\int_{0}^{p} \phi(\mathrm{u}) d u \text { and } \mathrm{p} \in[0,1]
$$

Through Equation (2), Dennerberg (1994) successfully proves the connection between a concave distortion function with a spectral risk measure. Thus, such concave distortion function has four properties of a SRM function.

This paper derives spectral risk measures

1 Acerbi (2002, 2004) considered this condition as decreasingness. However, he was dealing with distributions in which loss outcomes were negative rather than positive as in this paper. This difference is actually negligible. 
from the following three distortion functions:

Dual-power functions: $f(p)=1-p^{\gamma}$

Proportional hazard transform function:

$f(p)=(1-p)^{\frac{1}{\gamma}}$

Wang's distortion function:

$$
g_{\alpha}(p)=\Phi\left[\Phi^{-1}(1-p)+\alpha\right]
$$

where:

$p$ : probability

$f(p)$ : the distortion function of $p$

$\Phi$ : the standard normal cumulative density function

$\alpha, \gamma:$ risk aversion coefficient

$g_{\alpha}(p)$ : the distortion operator of $\mathrm{p}$ and $\alpha$

It should be noted that the distortion functions are defined such that the loss outcomes are expressed in positive numbers whereas loss is expressed in negative number in insurance context.

According to Henryk and Silvia (2007), a concave distortion risk measure is considered a spectral risk measure (i.e. $\rho_{f}(X)=M_{\phi}$ ) if the spectral risk function equates the first derivative of the distortion function $f(p)$ (i.e. $f^{\prime}(p)$ ). Given the distortion functions above, the respective risk aversion function $(\phi(p))$ are as follows:

Dual-power measure: $\phi(\mathrm{p})=\gamma p^{\gamma-1}$

Proportional hazard measure:

$\phi(\mathrm{p})=\frac{1}{\gamma}(1-p)^{\frac{1}{\gamma}-1}$

Wang's distortion measure:

$$
\phi(\mathrm{p})=e^{\left[-\alpha \Phi^{-1}(1-p)-\frac{\alpha^{2}}{2}\right]}
$$

Among the three, Wang's measure increases more rapidly than the proportional hazard measure. Accordingly, investors satisfying Wang's risk aversion function are more riskadverse than those with proportional hazard measure. According to Acerbi (2002), all of the three measures satisfy the first two conditions for an admissible risk aversion function. However, the limit of lambda needs to be defined so that the third condition is also satisfied.

Dual-power measure

$$
\phi(\mathrm{p})=\gamma p^{\gamma-1} \rightarrow \phi^{\prime}(\mathrm{p})=\gamma(\gamma-1) p^{\gamma-2} .
$$

As $\phi(\mathrm{p})>0$ so that $\gamma>1$

Figure 1 illustrates the case where $\gamma$ equal to 1.5 or 5 . Higher loss is assigned with higher weight. An increase in lambda is translated into higher level of risk aversion, and even higher weight is assigned to higher loss. Thus, $\gamma \in[0,5]$ satisfies the third condition of "weak increasingness."

Proportional hazard measure:

$$
\begin{aligned}
& \phi(\mathrm{p})=\frac{1}{\gamma}(1-p)^{\frac{1}{\gamma}-1}, \text { therefore } \phi^{\prime}(\mathrm{p})= \\
& \frac{1}{\gamma}\left(1-\frac{1}{\gamma}\right)(1-p)^{\frac{1}{\gamma}-2} \\
& \text { As } \phi^{\prime}(\mathrm{p})>0, \text { so that } \gamma>1
\end{aligned}
$$

Figure 2 depicts the behavior of proportional hazard transform weighting function for $\gamma=$ 1.5 and $\gamma=10$, where higher loss is consistently assigned higher weight. When $\gamma=$ 10 , the weight attached to higher loss rise more rapidly, compared to the weight rise when $\gamma=$ 1.5. Thus, this measure also meets the third condition of "weak increasingness" for $\gamma \in$ $[1.5,10]$. 


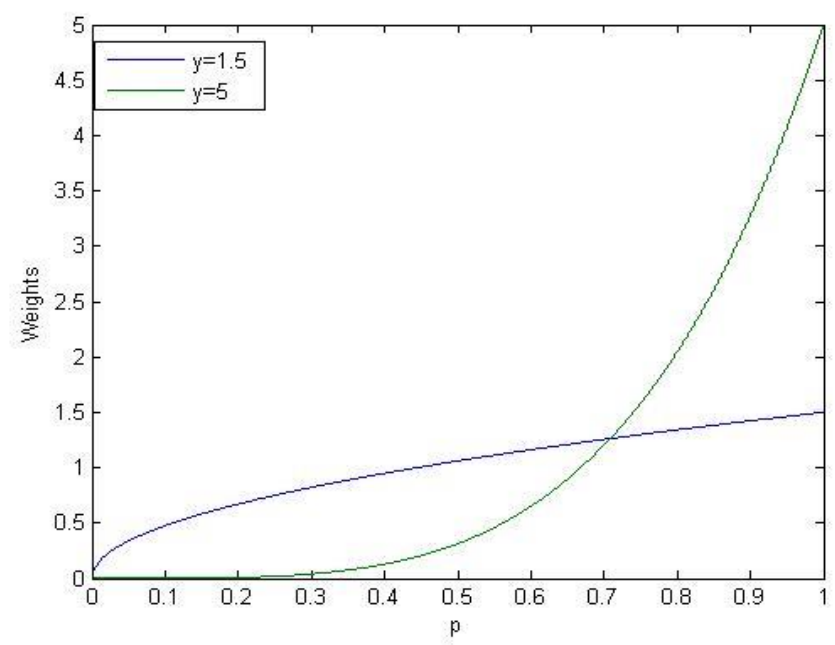

Figure 1. Plot of the weighting function derived from dual power function against cumulative probability with risk aversion coefficient equal to 1.5 and 5

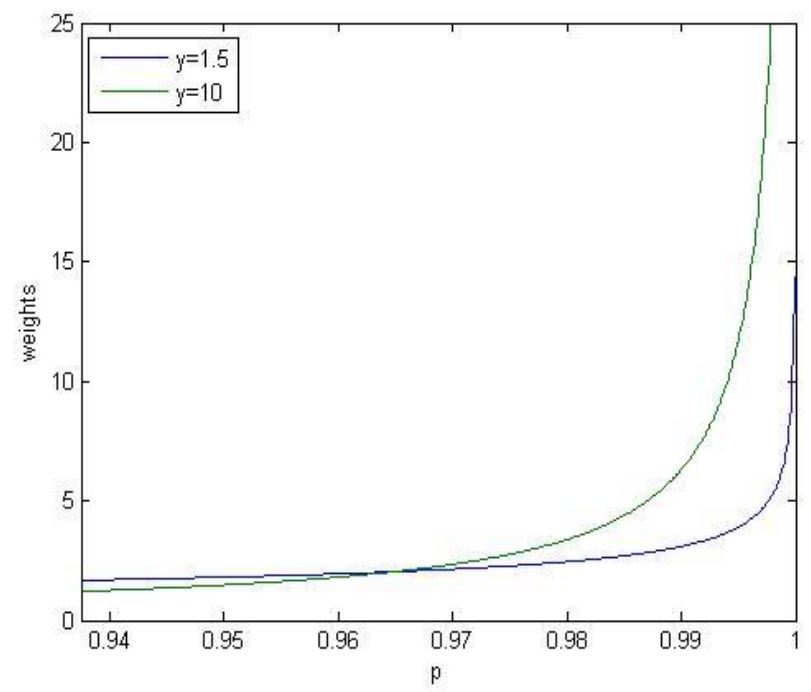

Figure 2. Plot of the weighting function derived from proportional hazard transform against cumulative probability with risk aversion coefficient equal to 1.5 and 10

Wang's distortion measure:

$\phi(\mathrm{p})=e^{\left[-\alpha \Phi^{-1}(1-p)-\frac{\alpha^{2}}{2}\right]}$, therefore $\phi^{\prime(\mathrm{p})}=$ $e^{\left[-\alpha \Phi^{-1}(1-p)-\frac{\alpha^{2}}{2}\right]} \times \frac{\alpha p}{\sqrt{2 \pi}} \times e^{\frac{-x^{2}}{2}}$

The special feature of Wang's measure is $\phi^{\prime}(\mathrm{p})>0$ for all $p \in[0,1]$.
Similar to the other measures, Wang's consistently satisfies the third condition of an admissible risk aversion function by assigning higher weight to higher loss. Notably, the assigned weight rises exponentially in the 99.5th to 100 th percentile when $\propto=5$, compared with a less dramatic increase in weight in the same 


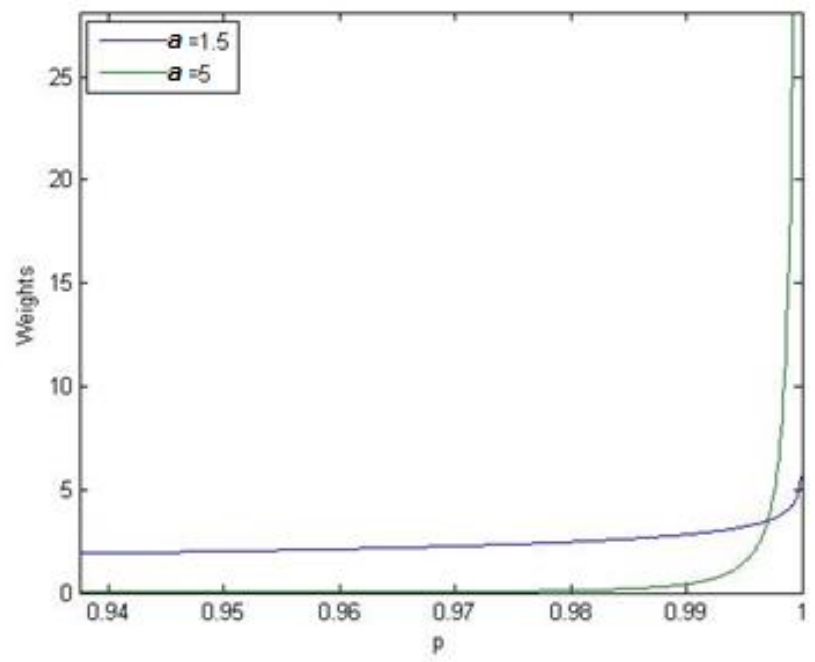

Figure 3. Plot of the weighting function derived from Wang's distortion function against cumulative probability with risk aversion coefficient equal to 1.5 and 5

percentile range when $\propto=1.5$.

It is essential to note that even though weights attached to higher losses rise proportionally to higher lambda, it is only applicable to a certain interval of lambda, depending on which measure is used and number of slides $\mathrm{p}$ is broken into. In addition, for these three measures, $M_{\phi} \rightarrow \int_{0}^{1} q_{p} d p$ as $\propto \rightarrow 1$, so the spectral risk measure approaches the mean of the loss distribution as $\gamma$ approaches 1 . Dowd et al. (2008) noted that this is an abnormal feature of spectral risk measure. Nonetheless, this paper rules out the special case that $\alpha=1$ (i.e. the spectral risk measure never equates the mean of the loss distribution).

\section{Methods}

\subsection{Estimating spectral risk measures}

Solving SRM following Equation (1) involves integration which would need executing numerically rather than analytically. To solve this integration four numerical quadrature methods to be considered consist of Trapezoid rule, Simpson's rule, Niederreiter and Weyl quasi Monte Carlo (Borse, 1997). These four methods, estimating the integral by breaking the probability of the whole loss distribution into $\mathrm{N}$ slides, would give the estimates to converge on their true values as $\mathrm{N}$ becomes larger. Dowd et al. (2007) indicated that the first two methods lead to more accurate results than the last two in the event of larger N. Dowd and his colleagues also proved that Simpson rule is marginally better than Trapezoid rule in the estimation of the integral. For this reason Simpson's rule is adopted in this study.

This paper divides $p$ horizon into 9998 intervals or 9999 points, each of which represents a quantile, and each quantile is calculated as the expected return of the portfolio minus the product of standard normal cumulative density function with the respective $p$ and standard deviation of the portfolio (i.e. a quantile $\left.q_{p}=\mu-Z_{p} \times \sigma\right)$. Every quantile is then assigned a weight defined by the weighting function $\phi(p)$. 


\subsection{Estimating confidence intervals for spectral risk measures}

This paper runs both parametric and nonparametric bootstrap. The bootstrap based on the most recent portfolio data reflects the future portfolio movements better than historical data since the latter is a bias reflection of the future. The procedure of estimation is as follows:

With $\mathrm{N}$ slides set, in each of $\mathrm{m}$ bootstrap trials, simulate a set of $\mathrm{N}$ losses from assumed distribution on the basis of Wiener process. If a parametric bootstrap is used, the simulated losses are ranked from lowest to highest to acquire a set of $\mathrm{N}$ quantiles. If a non-parametric bootstrap is used, we calculate each quantile with the formula $q_{p}=\mu-Z_{p} \times \sigma$, based on the law of large number and the central limit theorem. With each quantile corresponding to each confidence level, we multiply such quantile by the respective weight to obtain SRM.

The above process is reiterated $b$ times to yield the confidence interval from a distribution of simulated SRM.

This paper adopts the simulation bootstrap method since it is built upon a superior theoretical basis and especially suitable for such a volatile market as Vietnam. The model accuracy is determined by the number of slides and bootstrap setting; higher specification yields more accurate results. However, we implement 9998 slides and 1000 bootstrap trials to balance the processing time and the model accuracy. We then simulate portfolio returns to follow normal distribution and estimate non-parametric quantiles, upon which the spectral risk measure can be computed. After that, we exemplify SRM when the risk aversion coefficient equal 25 and 100 and apply $95 \%$ confidence interval. In the next step we take the next 14 consecutive returns of the portfolios in the respective 14 consecutive trading days, each corresponding with SRM with the risk aversion coefficient equaling 25, 100, and 200 and simulated from the past 100 trading day returns. Finally, we make a comparison between each estimated SRM and real loss. A similar process is implemented with $\mathrm{VaR}$ and ES at confidence levels of $95 \%$ and $99 \%$, and a comparison of results is used to demonstrate the advantage of SRM.

\subsection{Data}

This study has been performed on a pool of two portfolios, namely S\&P500 and VNIndex under normal and volatile market conditions. Two portfolios reflect two different markets with distinctive features. US stock market is one of the most mature markets worldwide while Vietnamese stock market is still in its infant stage. Two market conditions chosen are predicated on daily movement of two portfolios. Risk measures are carried out from two sets of data. The first comprises 100 index prices of 100 consecutive trading days in 2015. This set is used for bootstrapping and subsequently estimating confidence interval of simulated SRM. Figure 4 illustrates that the standard deviation of VNIndex portfolio returns in volatile market is remarkably higher than that in normal market. Additionally, Vietnam stock market witnesses both upside and downside in the volatile market while the upside seems to prevail in normal condition. S\&P500 displays the same behavior as VNIndex, but substantially less volatile in both conditions.

The second set consists of more than 750 consecutive trading days including 100 prices above, utilized for plotting SRM curve against coefficient of risk aversion and for making comparisons among SRM, expected shortfall, and value-at-risk. Under volatile market condition, two portfolios' prices are collected to examine the accuracy of SRM as a reflection of genuine risk of the portfolios. Thus, just the most recent part of the set is from volatile market condition, and the remaining part is from normal 
market condition. Specifically, Figure 5 first half. Besides, both sets are not apparently demonstrates that the second half of the set normal distributed due to high values of kurtosis illustrates stronger portfolio movement than the and skewness.

\section{Table 1}

Standard deviation of VNIndex and S\&P500 in two conditions in the first dataset

\begin{tabular}{lcc}
\hline Standard Deviation & In normal market condition & In volatile market condition \\
\hline VNIndex & $1.21 \%$ & $2.07 \%$ \\
S\&P500 & $0.79 \%$ & $1.60 \%$ \\
\hline
\end{tabular}
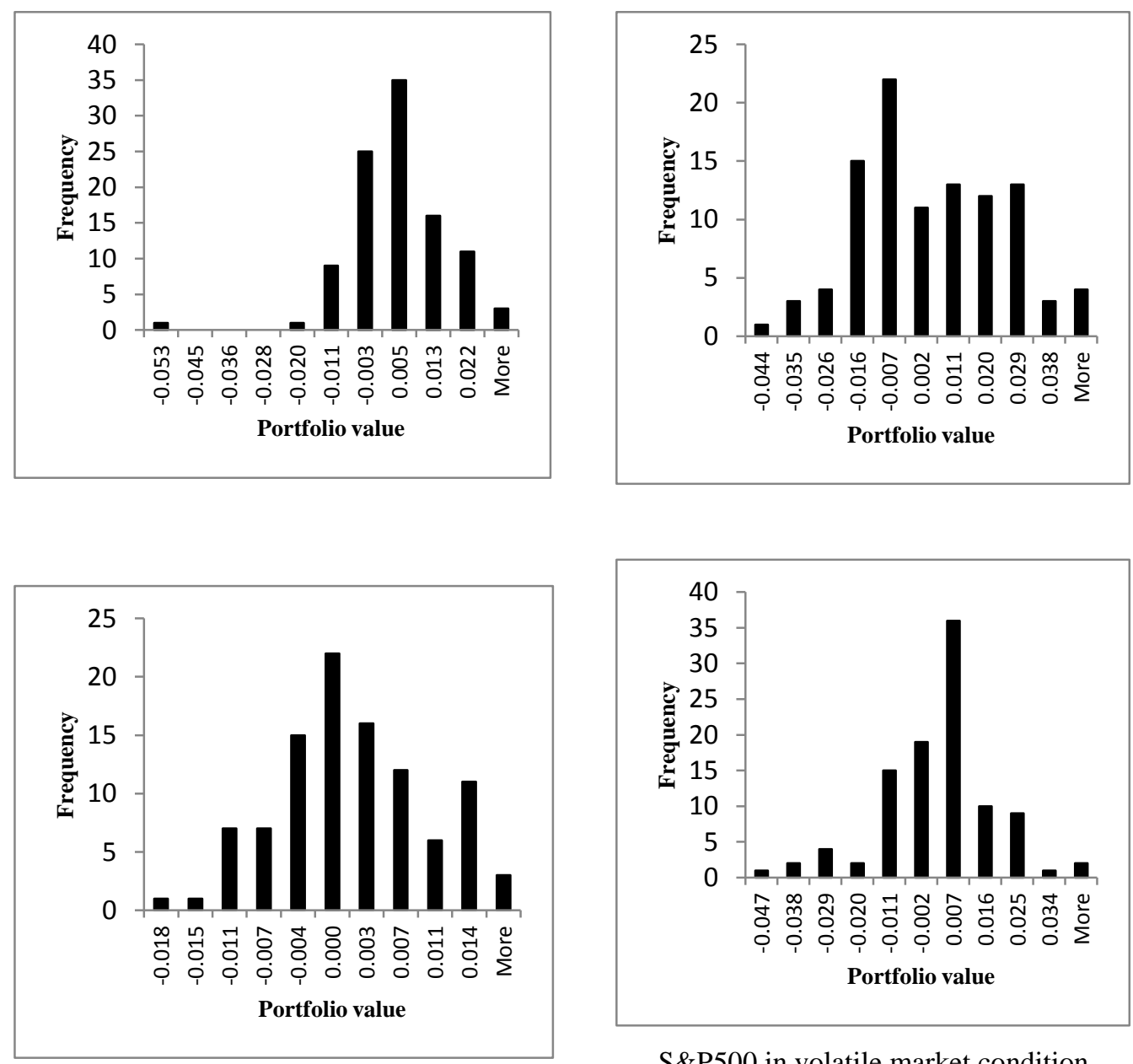

S\&P500 in volatile market condition

S\&P500 in normal market condition

Figure 4. Charts of the first dataset consisting of 100 trading returns of VNIndex and S\&P500 under normal and volatile market condition, collected from Stoxplus and Bloomberg 
Table 2

Standard deviation of VNIndex and S\&P500 in two conditions in the second dataset

\begin{tabular}{lcc}
\hline Standard Deviation & In normal market condition & In volatile market condition \\
\hline VNIndex & $1.08 \%$ & $1.94 \%$ \\
S\&P500 & $0.75 \%$ & $0.90 \%$ \\
\hline
\end{tabular}

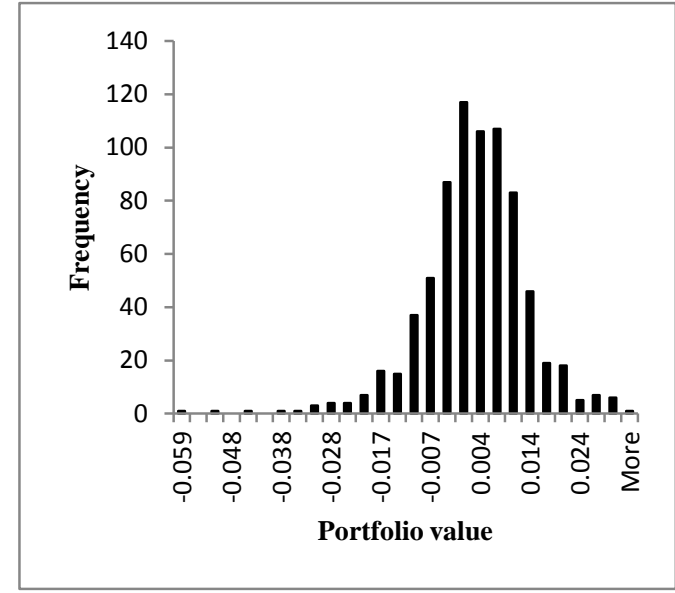

VNIndex in normal market condition

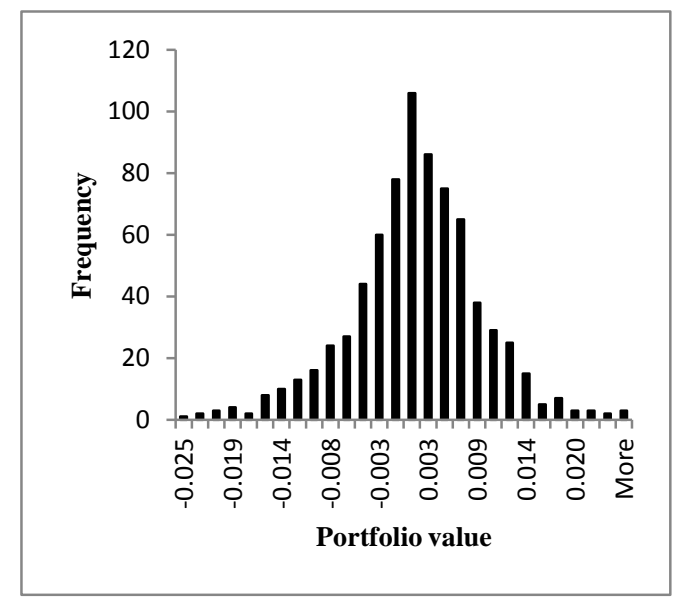

S\&P500 in normal market condition

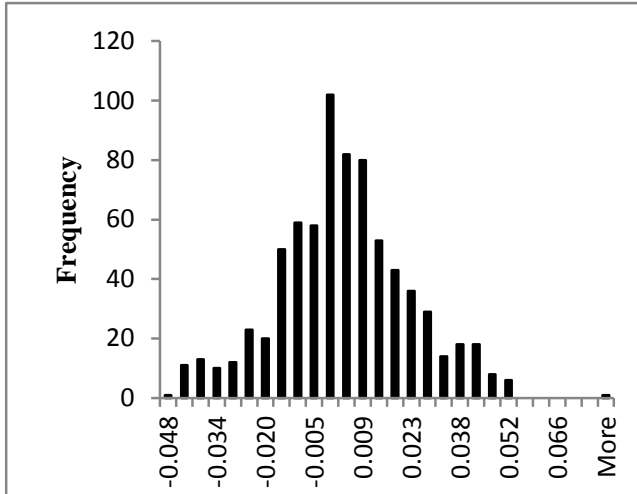

Portfolio value

VNIndex in volatile market condition

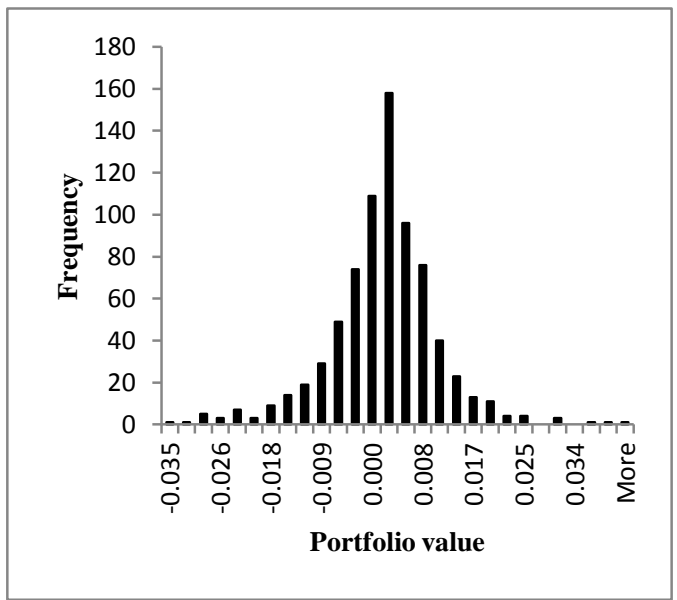

S\&P500 in volatile market condition

Figure 5. Charts of the second dataset consisting of 100 trading returns of VNIndex and S\&P500 under normal and volatile market condition, collected from Stoxplus and Bloomberg 


\section{Results and discussion}

\subsection{Results}

\section{Estimating spectral risk measures}

The following figures present the simulation results of dual power measure, the proportional

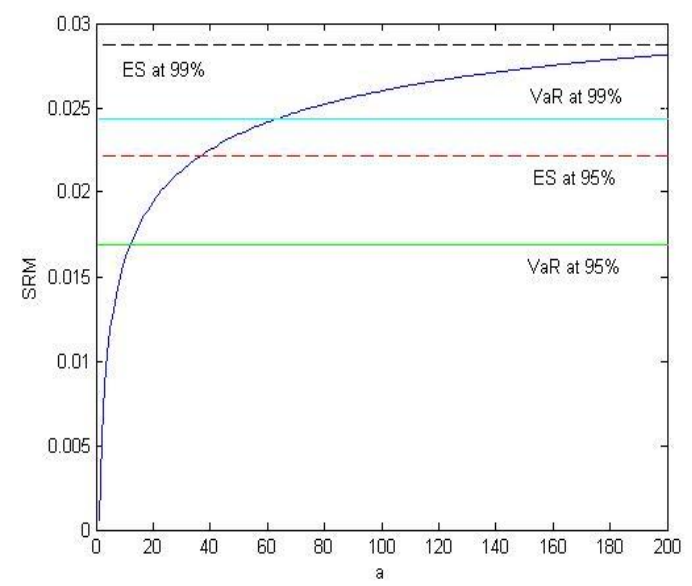

VNIndex in normal market condition

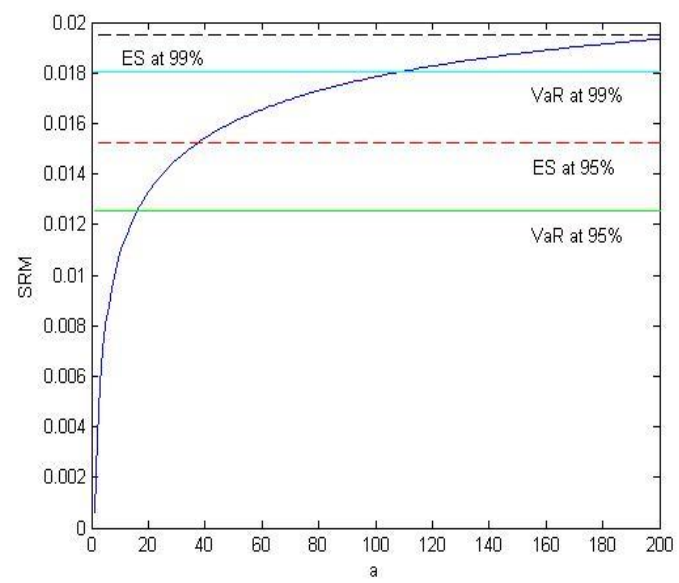

S\&P500 in normal market condition hazard measure and Wang's measure under four scenarios: VNIndex and S\&P500 Index under normal and volatile conditions.

\section{Dual power measure}

The SRM formula for dual power measure is $M_{\phi}=\int_{0}^{1} \gamma p^{\gamma-1} q_{X}(p) d p$.

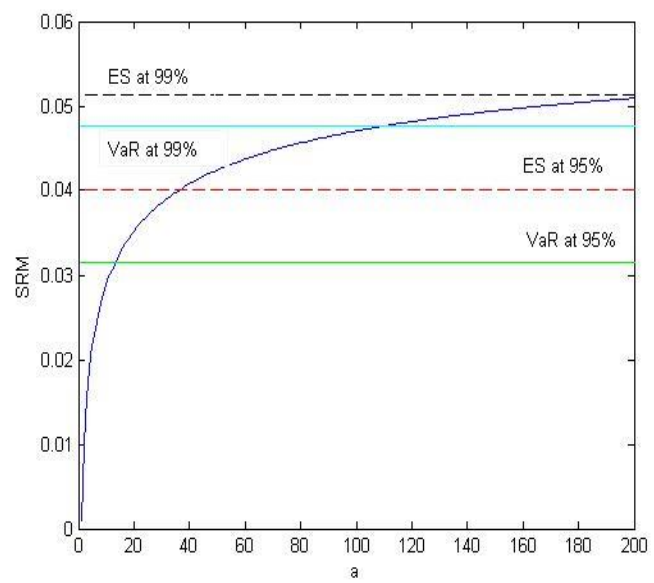

VNIndex in volatile market condition

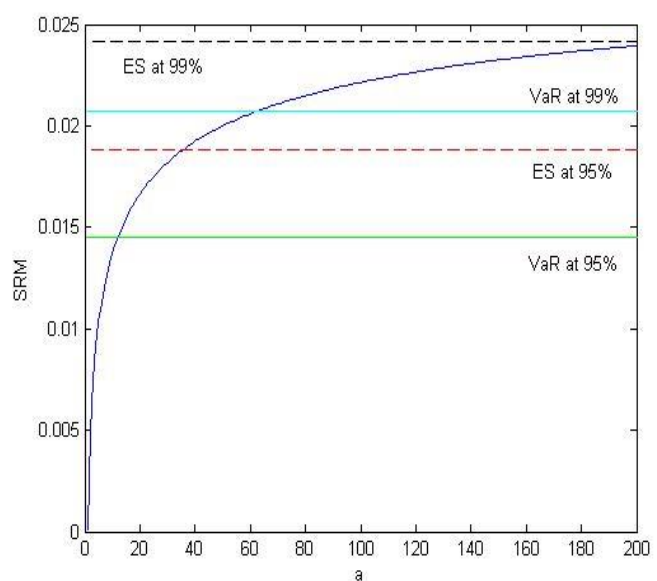

S\&P500 in volatile market condition

Figure 6. Plots of SRM curves of two portfolios against risk aversion coefficients under two different market conditions, based on Dual Power Measure and derived from the second dataset, using Matlab 2014a 
In all four scenarios, SRM rises rapidly when risk aversion level $(a)$ reaches up to 30 , but then the momentum is reduced when $a$ increases beyond 30. The curves are consistent with the risk aversion behavior in that the risk perception hikes when the risk aversion increases, but the marginal growth of SRM declines after a critical level of risk aversion (i.e. when $a=30$ ). Figure 6 demonstrates that the SRM measure is more flexible while VaR and ES are more conservative with various levels of risk aversion. Particularly, SRM overwhelms both VaR and ES when the

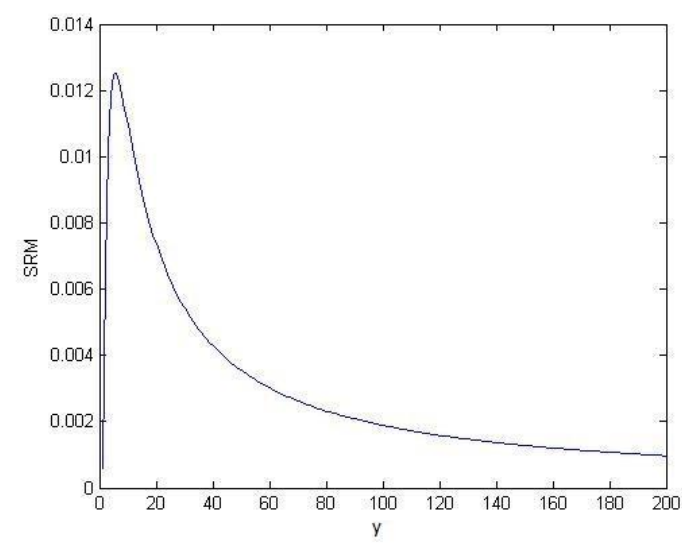

VNIndex in normal market condition

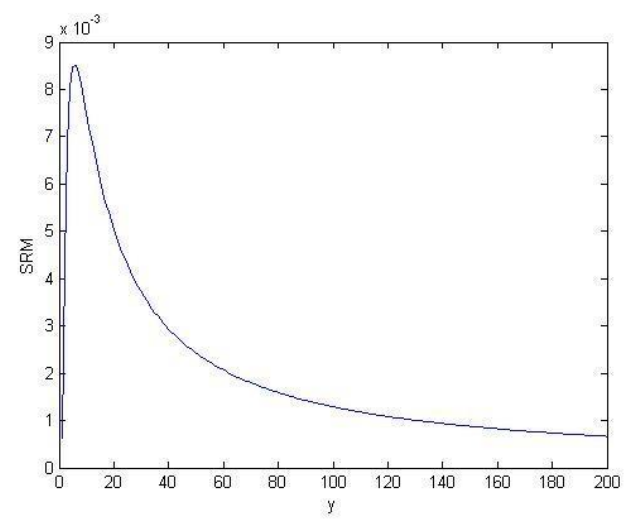

S\&P500 in normal market condition aversion level is under 30. SRM is also a better measure than ES for all levels of aversion.

\subsection{Proportional hazard measure}

The proportional hazard measure is based on the proportional hazard transform to build the following spectral risk aversion function:

$$
M_{\phi}=\int_{0}^{1} \frac{1}{\gamma}(1-p)^{\frac{1}{\gamma}-1} q_{X}(p) d p
$$

It is plotted with two portfolios under two different market conditions as below:

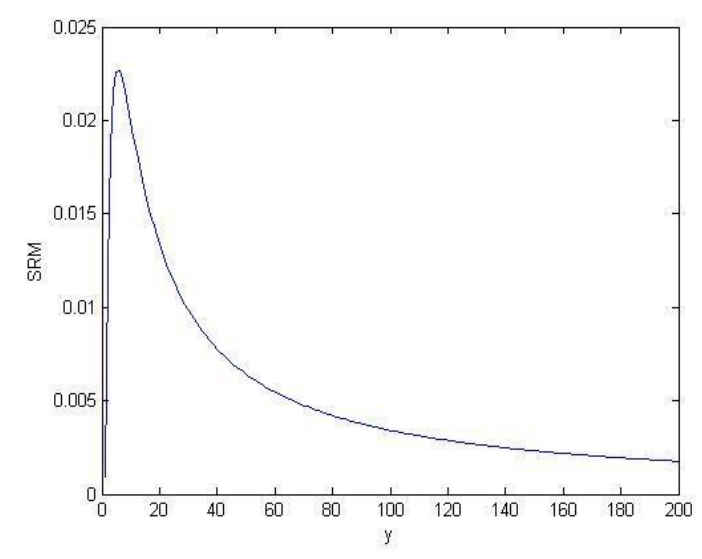

VNIndex in volatile market condition

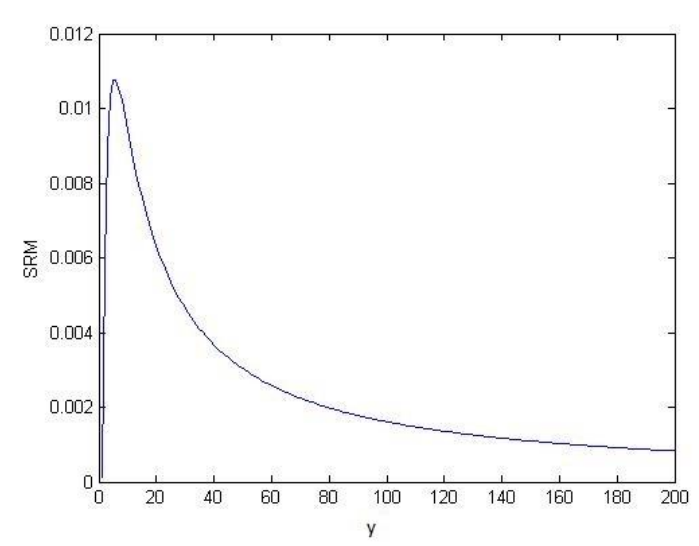

S\&P500 in volatile market condition

Figure 7. Plots of SRM curves of two portfolios against risk aversion coefficients under two different market conditions, based on Proportional Hazard Measure and derived from the second dataset, using 
Figure 7 illustrates the proportional hazard measure for VNIndex and S\&P500 indices under normal and volatile market conditions. $M_{\phi}$ rises exponentially as $a$ increases up to 10 but then falls downhill toward zero level. Although SRM calculated in the proportional hazard transform satisfies three conditions of the risk aversion function and it is consistently well below VaR and ES at $95 \%$ confidence level in all four settings ${ }^{3}$, this measure is only applicable when aversion level $a$ is in the range between 0 and 10 since SRM $\left(M_{\phi}\right)$ corresponding to this range is an increasing function. Meanwhile SRM outside such range is a decreasing function of $a$, which renders this measure ineffective. The rising part of the SRM curve will be marginally prolonged if $p$ is divided into more slides than the existing number (9999), making proportional hazard measure more efficient.

\section{Wang's measure}

The Wang's risk measure derived from Wang's distortion function is computed as below:

$$
M_{\phi}=\int_{0}^{1} e^{\left[-\alpha \Phi^{-1}(1-p)-\frac{\alpha^{2}}{2}\right]} q_{X}(p) d p
$$

The SRM under Wang's distortion function in all four settings is resisted by the ES at $95 \%$, indicating the advantage of Wang's SRM over ES measure. It also demonstrates the flexibility of SRM over $\mathrm{VaR}$ in that $\mathrm{VaR}$ is conservative regardless of the variety of risk aversion coefficient $a$, while SRM is lower than VaR (95\%) when $a \in(0,20)$ and higher than the same VaR when $a \in(20,30)$. However, Wang's SRM is only applicable when it is an increasing function of $a$, which is similar to proportional hazard measure. The rising part of SRM curves is also marginally prolonged when $p$ horizon is divided into larger number of slides, resulting in a more efficient measure of portfolio's risk.

Estimating confidence intervals for spectral risk measures

Table 3 further demonstrates the flexibility of SRM, which shows the SRM under Dual Power weighting function with three different levels of risk aversion. With SRM, the more risk-adverse the investor, the higher expected loss, whereas VaR and ES use confidence level (95\% or 99\%) to account for risk aversion in that a highly riskadverse investor chooses $99 \%$ and a less riskadverse investor chooses $95 \%$. Both confidence levels are popular benchmarks and often reported as a rule of thumb. VaR and ES entail no common levels such as $93.7 \%$ or $91.2 \%$, hence lacking flexibility compared to the continuous level of risk aversion $\propto$. Moreover, Table 4 illustrates that SRM is better than the traditional $\mathrm{VaR}$ in volatile market condition, while being as good as VaR and ES measures in normal condition.

\footnotetext{
${ }^{3}$ The VaR and ES at 95\% confidence level is too high above the SRM line, hence not being drawn in figure 7.
} 


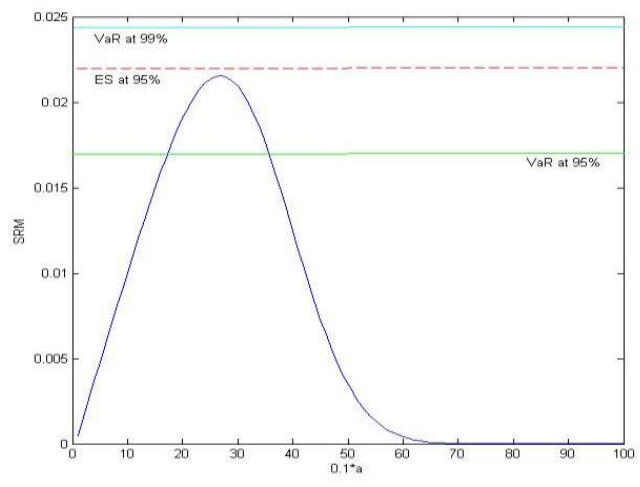

VNIndex in normal market condition

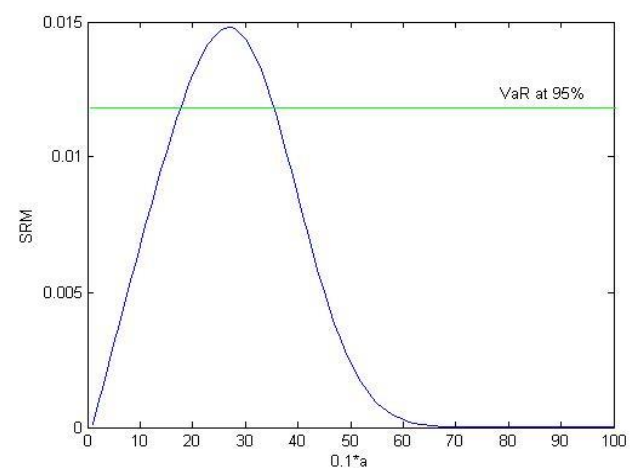

S\&P500 in normal market condition

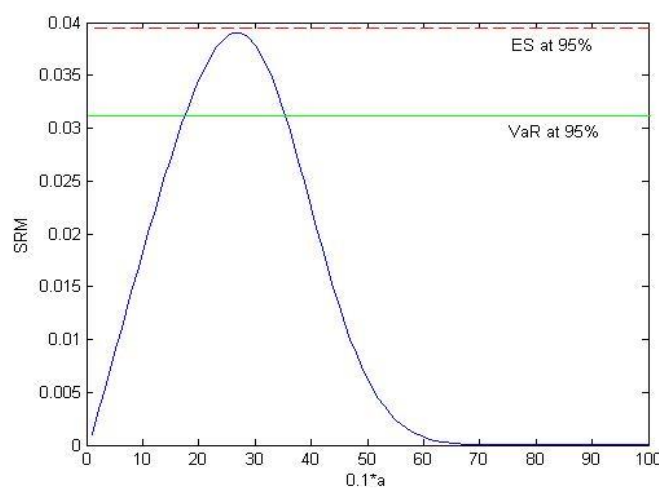

VNIndex in volatile market condition

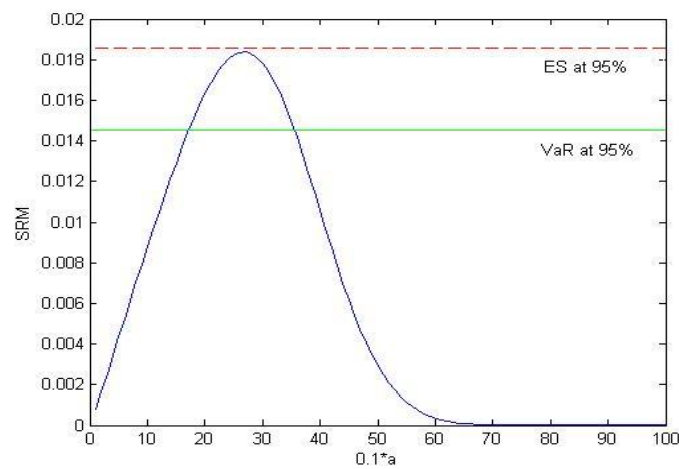

S\&P500 in volatile market condition

Figure 8. Plots of SRM curves of two portfolios against risk aversion coefficients under two different market conditions, based on Wang's measure and derived from the second dataset

\section{Table 3}

The confidence intervals of two portfolios under normal and volatile market condition

\begin{tabular}{cccccc}
\hline \multicolumn{2}{c}{ Portfolio } & \multicolumn{2}{c}{ VNIndex } & \multicolumn{2}{c}{ S\&P500 } \\
\hline \multicolumn{2}{c}{ Market condition } & Normal & Volatile & Normal & Volatile \\
\hline \multirow{2}{*}{ SRM } & $\mathrm{a}=25$ & {$[0.0232,0.0233]$} & {$[0.0413,0.0414]$} & {$[0.0151,0.0152]$} & {$[0.0325,0.0326]$} \\
& $\mathrm{a}=100$ & {$[0.0294,0.0295]$} & {$[0.0519,0.0520]$} & {$[0.0191,0.0192]$} & {$[0.0407,0.0408]$} \\
& $\mathrm{a}=200$ & {$[0.0318,0.0319]$} & {$[0.0561,0.0562]$} & {$[0.0207,0.0208]$} & {$[0.0439,0.0440]$} \\
\multirow{2}{*}{ VaR } & $95 \%$ & {$[0.0195,0.0196]$} & {$[0.0350,0.0351]$} & {$[0.0127,0.0128]$} & {$[0.0277,0.0278]$} \\
& $99 \%$ & {$[0.0277,0.0278]$} & {$[0.0491,0.0492]$} & {$[0.0180,0.0181]$} & {$[0.0385,0.0386]$} \\
\multirow{2}{*}{ ES } & $95 \%$ & {$[0.0215,0.0216]$} & {$[0.0441,0.0442]$} & {$[0.0151,0.0152]$} & {$[0.0311,0.0312]$} \\
& $99 \%$ & {$[0.0280,0.0282]$} & {$[0.0551,0.0552]$} & {$[0.0194,0.0195]$} & {$[0.0420,0.0422]$} \\
\hline
\end{tabular}

Notes: The intervals are measured with SRM, VaR, and ES with various parameters (SRM is measured with dual power weighting function). For each method, the reported value is the expected range of loss for SRM, VaR, and ES. 
Table 4

Number of cases where the loss in reality exceeds the benchmar in SRM, VaR, and ES

\begin{tabular}{ccccccccc}
\hline & \multicolumn{3}{c}{ VaR } & \multicolumn{2}{c}{ ES } & \multicolumn{3}{c}{ SRM } \\
\cline { 3 - 9 } Market condition & $95 \%$ & $99 \%$ & $95 \%$ & $99 \%$ & $\mathrm{a}=25$ & $\mathrm{a}=100$ & $\mathrm{a}=200$ \\
\hline \multirow{2}{*}{ S\&P500 } & Normal & 0 & 0 & 0 & 0 & 0 & 0 & 0 \\
& Volatile & 2 & 0 & 1 & 0 & 1 & 0 & 0 \\
\multirow{2}{*}{ VNIndex } & Normal & 0 & 0 & 0 & 0 & 0 & 0 & 0 \\
& Volatile & 4 & 0 & 3 & 0 & 3 & 0 & 0 \\
\hline
\end{tabular}

\subsection{Discussion}

SRM curve entails different shapes for each distortion function (i.e. dual power, proportional hazard transform, and Wang's), and each curve is suitable for the investor with different risk appetites. Our results demonstrate that dual power function results in the increasing SRM function while the other two distortion functions lead to parabolic shapes of SRM curve. However, only the increasing portion of the parabola in the latter two methods is applicable to risk measurement since it is consistent with the principle that higher weight is attached to higher loss and that lower weight is assigned to lower loss. Thus, dual power measure can be used for full spectrum of risk aversion coefficient $a$ while Wang's and proportional hazard functions are limited to a certain level of risk aversion where the respective SRM is an increasing function.

One noteworthy feature of SRM is that this measure, based on bootstrapping method, is more conservative than VaR and ES, which are measured upon historical data. More importantly, this paper finds that SRM barrier is less frequently penetrated than VaR and ES in volatile market conditions, which is consistent with the theoretical framework.

\section{Conclusion}

This paper has examined spectral risk measure derived from three distortion functions. It has been found that even though three respective weighting functions satisfy all the conditions of a risk aversion function, they do not necessarily create a meaningful risk measure for risk-averse investors. This indicates a lack of another requirement for selecting appropriate functions to transform to spectral risk measures. We also discover that when investigating each risk measure curve, users should be concerned about the range of risk aversion coefficient so that the derivatives of risk aversion function along with the coefficient is above zero and on the use of estimation rule, such as Simpson's, and also should consider the number of slides of cumulative probability that may give rise to a gain in the risk measure but takes more time to estimate.

Among the three distortion functions gauged, dual power measure may be the most sensible measure with desirable properties. The risk measure not only meets requirements of a spectral risk measure, but also smoothly increases along with risk aversion coefficient of users. The measure is more flexible and useful 
for the users than value-at-risk and expected shortfall. The measure would be even better at reflecting the true portfolio risk if combined with simulation approach in order to keep the measure updated with recent information. Meanwhile, proportional hazard and Wang's measure, despite satisfying conditions of an expected weighting function of spectral risk measure, may not be useful for some group of risk averse users as their peak is not as high as VaR and ES at confidence level of $99 \%$, which is presently within risk appetite of some users like commercial banks.

The paper also has exhibited no major differences when spectral risk measure is employed to measure portfolio risk in Vietnam and in the U.S. The discrepancy is resulted from volatility of each portfolio, rather than the essence of the model. Provided that users are risk-averse, they can take advantage of spectral risk measures with respective weighting functions matching their levels of risk aversion. SRM is especially suitable for Vietnam bankers who manage portfolios or risky loans since it is flexible in the consideration of various borrower's risk aversion, compared to $\mathrm{VaR}$ and ES, which only account for risk aversion with the change of confidence level (i.e. $95 \%$ or $99 \%$ ).
We also demonstrate that distortion functions, despite satisfying Acerbi's (2002, 2004) conditions of "well-behaved" risk measure, requires extra conditions to control for the abnormality of SRM curves when the SRM is the decreasing function of risk aversion coefficient (a).

Commercial bankers may take advantage of spectral risk measure in volatile market conditions since this measure seems to outperform the traditional $\mathrm{VaR}$ and ES. In addition, SRM not only entails a cautious approach to risk measure, which is consistent with banker merit, but it also facilitates the flexible change in the risk tolerance level to match fluctuating economic conditions.

Further research may focus on other distortion functions to extend their applicability in risk measuring. Potential topic may also refer to the examination of various specifications of distortion function so that the resulting SRM curve is suitable for a particular group of riskadverse investors.

\section{Acknowledgement}

This study is an outcome of the research project on "Firm's behavior and adaptation in the context of international integration," which is sponsored by Foreign Trade University

\section{References}

Abad, C., \& Iyengar, G. (2015). Portfolio selection with multiple spectral risk constraints. Retrieved April 5, 2016 from https://arxiv.org/abs/1410.5328

Acerbi, C. (2002). Spectral measures of risk: A coherent representation of subjective risk aversion. Journal of Banking and Finance, 26, 1505-1518.

Acerbi, C. (2004). Coherent representations of subjective risk aversion. In G. Szegö (Ed.), Risk measures for the 21 st century (pp. 147-207). Wiley, NY.

Adam, A., Houkari, M., \& Laurent, J. P. (2007). Spectral risk measures and portfolio selection. Journal of Banking \& Finance, 32(9), 1870-1882.

Artzner, P., Delbaen, F., Eber, J. M., \& Heath, D. (1997). Thinking coherently. Risk, 10(November), 68-71.

Artzner, P., Delbaen, F., Eber, J. M., \& Heath, D. (1999). Coherent measures of risk. Mathematical Finance, 9, 203-228. 
Borse, G. J. (1997). Numerical methods with MATLAB: A resource for scientists and engineers. PWS Publishing Company, Boston.

Cotter, J., \& Dowd, K. (2006). Extreme spectral risk measures: An application to futures clearinghouse margin requirements. Journal of Banking and Finance, 30, 3469-3485.

Cotter, J., \& Dowd, K. (2006). Extreme spectral risk measures: An application to futures clearinghouse margin requirements. Journal of Banking and Finance, 30, 3469-3485.

Denneberg, D. (1994). Non-additive measure and integral. Kluwer Academic Publishers, Dordrecht, Germany.

Dowd, K., \& Cotter, J. (2007). Exponential spectral risk measure. Retrieved March 25, 2016 from https://arxiv.org/ftp/arxiv/papers/1103/1103.5409.pdf

Dowd, K., Cotter, J., \& Sorwar, G. (2008). Spectral risk measures: Properties and limitations. Journal of Financial Services Research, 34, 61-75.

Global Association of Risk Professionals (GARP). (2016). Financial risk management, market risk measurement and management (5th Ed.). Pearson Education, Inc.

Grootveld, H., \& Hallerbach, W. G. (2004). Upgrading value-at-risk from diagnostic metric to decision variable: A wise thing to do? In G. Szegö (Ed.), Risk measures for the 21 st century (pp. 33-50). Wiley, NY.

Guegan, D., \& Hassani, B. (2014). Distortion risk measures or the transformation of unimodal distributions into multimodal functions. Retrieved April 1, 2016 from http://centredeconomiesorbonne.univ-paris1.fr/

Gzyl, H., \& Mayoral, S. (2006). On a relationship between distorted and spectral risk measures. Retrieved April 1, 2016 from http://www.unav.edu/documents/29147/425795/wp1506.pdf

Markowitz, H. (1952). Portfolio selection. The Journal of Finance, 7(1), 77-91.

Overbeck, L. (2004). Spectral capital allocation. In A. Das (Ed.), Capital allocation. Risk Books, London.

Sereda, E. N., Bronshtein, E. M., Rachev, S. T., Fabozzi, F. J., Sun, W., \& Stoyanov, S. V. (2010). Distortion risk measures in portfolio optimization. In J. B. Guerard (Ed.), Handbook of portfolio construction (pp. 649-673). Springer, Boston, MA.

Sriboonchitta, S., Nguyen, H. T., \& Kreinovich, V. (2010). How to relate spectral risk measures and utilities. Departmental Technical Reports. Department of Computer Science. University of Texas.

Tasche, C., \& Acerbi, C. (2002). Portfolio optimization with spectral measures of risk. Working paper. Abaxbank, Italy.

Wang, S. S. (2000). A class of distortion operators for pricing financial and insurance risks. Journal of Risk and Insurance, 67(1), 15-36.

Yaari, M. E. (1987). The dual theory of choice under risk. Econometrica, 55(1), 95-115. 